\title{
Prognostic value of miR-1181 in non-small cell lung cancer and its regulatory effect on tumor progression
}

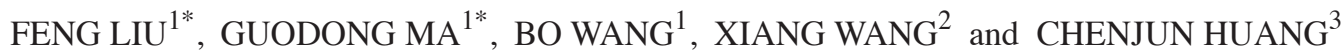 \\ ${ }^{1}$ Department of Chest Surgery, Nanjing Chest Hospital, Nanjing, Jiangsu 210029; \\ ${ }^{2}$ Department of Oncology, Xuzhou Central Hospital, Xuzhou, Jiangsu 221000; \\ ${ }^{3}$ Department of Thoracic Surgery, Jiangsu Province Hospital, Nanjing, Jiangsu 210029, P.R. China
}

Received September 28, 2020; Accepted March 19, 2021

DOI: $10.3892 / \mathrm{etm} .2021 .10719$

\begin{abstract}
Non-small cell lung cancer (NSCLC) is one of the most important causes of cancer-related death. miR-1181 has been reported to have roles in various cancer types and its function in the progression of NSCLC was investigated in the present study. A total of 118 patients with NSCLC were recruited and their tumor tissues were collected. The expression of miR-1181 in NSCLC tissues and cells was detected by reverse transcription-quantitative PCR. The prognostic value of miR-1181 was evaluated by Kaplan-Meier and Cox regression analysis and the roles of miR-1181 in cell proliferation, migration and invasion of NSCLC were analyzed by Cell Counting Kit- 8 and Transwell assays. miR-1181 was indicated to be upregulated in NSCLC tissues and cell lines, and to be associated with lymph node metastasis and the TNM stage of patients. Patients with high miR-1181 expression had a poorer prognosis than those with low miR-1181 expression. miR-1181 levels and TNM stage were determined to be two independent prognostic factors for NSCLC. In addition, overexpression of miR-1181 exerted enhancing effects on cell proliferation, migration and invasion of NSCLC, while its knockdown inhibited these cellular processes. In conclusion, upregulation of miR-1181 in NSCLC was associated with lymph node metastasis and TNM stage of patients and was indicative of poor prognosis. miR-1181 was indicated to exert promoting effects on cell proliferation, migration and invasion of NSCLC
\end{abstract}

Correspondence to: Dr Xiang Wang, Department of Oncology, Xuzhou Central Hospital, 199 South Jiefang Road, Quanshan, Xuzhou, Jiangsu 221000, P.R. China

E-mail: wangxiang7726@163.com

Dr Chenjun Huang, Department of Thoracic Surgery, Jiangsu Province Hospital, 300 Guangzhou Road, Nanjing, Jiangsu 210029, P.R. China

E-mail: huang_cj1975@163.com

*Contributed equally

Key words: non-small cell lung cancer, microRNA-1181, progression, prognosis cells and to be involved in tumor progression, providing novel insight for the development of biomarkers and therapies for NSCLC.

\section{Introduction}

Lung cancer is one of the most frequent causes of cancer-related death, with an incidence rate of 1.3 million cases per year and a 5-year survival rate of $<15 \%$ (1-3). Among the various subtypes of lung cancer, non-small cell lung cancer (NSCLC) is the most common type, which is mostly detected at an advanced stage (4). The low survival rate of NSCLC results from the limited efficacy of treatment and difficulties in the early detection of advanced disease (5). Targeted therapy and early detection are the most effective approaches to significantly reduce the mortality of patients with NSCLC. Therefore, it is urgently required to identify novel biomarkers that participate in the progression of NSCLC.

MicroRNAs (miRNAs/miRs) are short, noncoding RNAs with vital roles in the post-transcriptional regulation of gene expression and various biological processes (6). Dysregulated miRNAs have attracted special attention and have roles in tumorigenesis and cancer progression. For instance, miR-654-3p suppresses hepatocellular carcinoma progression (7). miR-188-5p promotes the proliferation and migration of gastric cancer cells (8). Accumulating evidence has indicated that certain miRNAs, such as miR-650, miR-195-5p and miR-30, regulate cell proliferation, migration, invasion and apoptosis in NSCLC (9-11). The deregulation of specific miRNAs may serve as a biomarker for the progression and prognosis of NSCLC.

miR-1181 was reported to be abnormally expressed in several cancer types, including ovarian cancer, pancreatic cancer, hepatocellular carcinoma and NSCLC, and was reported to be related to the development of these cancers (12-15). It was hypothesized that miR-1181 may have a vital role in the development and prognosis of NSCLC, but data on the function of miR-1181 in NSCLC have remained limited. In the present study, the expression of miR-1181 in NSCLC and adjacent tissues, as well as the association between miR-1181 expression and the clinical features and survival rate of patients were analyzed to evaluate the prognostic value of miR-1181. The function of miR-1181 in NSCLC 
cell proliferation, migration and invasion was also investigated through Cell Counting Kit-8 (CCK8) and Transwell assays.

\section{Materials and methods}

Specimens. Paired NSCLC and adjacent non-tumor tissues confirmed by at least three pathological experts were collected from 118 patients with NSCLC treated at Nanjing Chest Hospital (Nanjing, China) between January 2012 and December 2014. All patients provided written informed consent for participating in the present study and did not received any chemotherapy or radiotherapy prior to the surgery. The survival information of the patients with NSCLC was obtained in a 5-year follow-up survey. All tissues were frozen in liquid nitrogen immediately after collection and stored at $-80^{\circ} \mathrm{C}$. The present study was approved by the Ethics Committee of Nanjing Chest Hospital (Nanjing, China).

Cell culture and transfection. The human NSCLC cell lines A549, H1299, H2009 and NE18, as well as the normal human lung epithelial cell line BEAS-2B, were purchased from the American Type Culture Collection. Cells were cultured in RPMI-1640 medium (Invitrogen; Thermo Fisher Scientific, Inc.) containing 10\% FBS (Invitrogen; Thermo Fisher Scientific, Inc.), with maintenance at $37^{\circ} \mathrm{C}$ in a humidified atmosphere with $5 \% \mathrm{CO}_{2}$.

miR-1181 mimics $(50 \mathrm{nM}$; 5'-CCGUCGCCGCCACCC GAGCCG-3'), miR-1181 inhibitor (50 nM; 5'-CGGCUCGGG UGGCGGCGACGG-3') and corresponding negative control [mimics NC (5'-GGACCAAATCTCGAGATTTGG-3') and inhibitor NC (5'-UCUACUCUUUCUAGGAGGUUGUGA-3'); Guangzhou RiboBio Co., Ltd.] were transfected into cells using the Lipofectamine ${ }^{\circledR} 2000$ reagent (Invitrogen; Thermo Fisher Scientific, Inc.) at room temperature for $24 \mathrm{~h}$ according to the manufacturer's protocol to regulate the expression of miR-1181. After $24 \mathrm{~h}$ of transfection, the cells were ready for use in the subsequent experiments.

$R N A$ isolation and reverse transcription-quantitative $(R T-q)$ $P C R$. Total RNA was isolated from collected tissues and cell lines using TRIzol reagent (Invitrogen; Thermo Fisher Scientific, Inc.) according to the manufacturer's protocol and reverse-transcribed into complementary DNA at $42^{\circ} \mathrm{C}$ for 15 min and at $85^{\circ} \mathrm{C}$ for 2 min using a TaqMan MicroRNA Reverse Transcription Kit (Applied Biosystems; Thermo Fisher Scientific, Inc.). qPCR was performed with a SYBR-Green I Master Mix kit (Invitrogen; Thermo Fisher Scientific, Inc.) in the 7300 Real-Time PCR System (Applied Biosystems; Thermo Fisher Scientific, Inc.). The primer sequences were as follows: 5'- GTGCAGGGTCCGAGGTCAGAGCCACCT GGGCAATTTTTTTTTTTCGGCTC-3' for reverse-transcription, the forward primer was 5'-CCGGGCCGTCGC CGCCACCC-3', and the reverse primer was 5'-GTGCAGGGT CCGAGGTCA-3' for miR-1181 qPCR. U6 (forward, 5'-CTC GCTTCGGCAGCACA-3' and reverse, 5'-AACGCTTCACGA ATTTGCGT-3') was used as an internal control and the $2^{-\Delta \Delta C q}$ method was used to quantify the expression of miR-1181 (16). The thermocycling conditions were set as $94^{\circ} \mathrm{C}$ for $5 \mathrm{~min}$ and then 35 cycles of denaturation for $30 \mathrm{sec}$ at $94^{\circ} \mathrm{C}$, annealing for $30 \mathrm{sec}$ at $50^{\circ} \mathrm{C}$ and extension for $45 \mathrm{sec}$ at $72^{\circ} \mathrm{C}$.
CCK8 assay. According to the protocol for the CCK-8 Assay kit (Dojindo Molecular Technologies Inc.), the CCK8 assay was performed to analyze the proliferation of NSCLC cells. In brief, NSCLC cells at a density of $5 \times 10^{3}$ cells per well were seeded into 96-well plates in triplicates. After incubating for $0,24,48$ and $72 \mathrm{~h}$ at $37^{\circ} \mathrm{C}, \mathrm{CCK} 8$ reagent was added to each well, followed by further incubation for $4 \mathrm{~h}$ at $37^{\circ} \mathrm{C}$. The absorbance of each well at $450 \mathrm{~nm}$ was detected using a microplate reader (Thermo Fisher Scientific, Inc.) to evaluate the proliferation of NSCLC cells.

Transwell assay. NSCLC cells in serum-free medium were seeded into the upper wells of a 24-well Transwell chamber with $8-\mu \mathrm{m}$ pore size (Corning, Inc.) at $2 \times 10^{5}$ cells per well. Fresh culture medium with $10 \%$ FBS was added to the bottom chamber as the chemoattractant. After $48 \mathrm{~h}$ of incubation at $37^{\circ} \mathrm{C}$, cells on the upper surface of the membrane were removed and those that had transgressed to the bottom chamber were fixed with $4 \%$ paraformaldehyde for $20 \mathrm{~min}$ at room temperature and stained with $0.1 \%$ crystal violet for $10 \mathrm{~min}$ at room temperature. To assess cell invasion, the upper chamber was precoated with $0.5 \%$ Matrigel (BD Biosciences) at $37^{\circ} \mathrm{C}$ overnight. Migrated and invaded cells were counted by a light microscope (magnification, $\mathrm{x} 400$ ). The images were captured randomly from at least 5 fields and analyzed by ImageJ software (version 1.49; National Institutes of Health).

Luciferase reporter assays. Potential targets of miR-1181 were first predicted in silico using targetscan (http://www. targetscan.org/vert_72/) and then confirmed using a luciferase reporter assay. A fragment of the 3' untranslated region (3'UTR) of axin 1 (AXIN1) containing a prospective binding site for miR-1181 was amplified with the genomic DNA extracted from A549 cells using the same reverse transcription protocol as aforementioned used as a template and the AmpFISTR Identifiler PCR Amplification Kit (Applied Biosystems; Thermo Fisher Scientific, Inc.) according to the protocol. The amplification conditions were: Initial denaturation for $10 \mathrm{~min}$ at $95^{\circ} \mathrm{C}$, followed by 45 cycles of denaturation at $95^{\circ} \mathrm{C}$ for $10 \mathrm{sec}$, annealing for $20 \mathrm{sec}$ at $60^{\circ} \mathrm{C}$ and extension at $72^{\circ} \mathrm{C}$ for $20 \mathrm{sec}$. The amplified fragment was then inserted into the reporter vector pmirGLO (Promega Corporation) to construct the luciferase reporter plasmid AXIN1 wild-type (WT) according to previous studies $(17,18)$. Furthermore, its mutant (MT) sequence was also synthesized with mutations in the binding sites introduced using the QuikChange Site-Directed Mutagenesis kit (Stratagene; Agilent Technologies, Inc.) to construct the luciferase reporter plasmid AXIN1 MT. A549 cells were co-transfected with pGL3-AXIN1-WT (10 nM) or pGL-AXIN1-MT (10 $\mathrm{nM})$ and miR-1181 mimics $(50 \mathrm{nM})$, miR-1181 inhibitor $(50 \mathrm{nM})$ or negative controls $(50 \mathrm{nM})$ using Lipofectamine ${ }^{\circledR} 2000$ reagent (Invitrogen; Thermo Fisher Scientific, Inc.). The luciferase activity levels were analyzed by the Dual-Luciferase Reporter Assay Kit (Promega Corporation) with Renilla as the normalization control according to the manufacturer's protocol.

Statistical analysis. Statistical analyses were performed with GraphPad Prism 5.0 software (GraphPad Software, Inc.) and SPSS 20.0 software (IBM, Corp.). All values are expressed as 
A

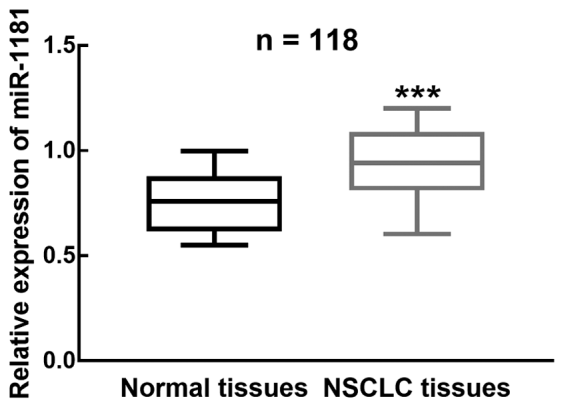

B

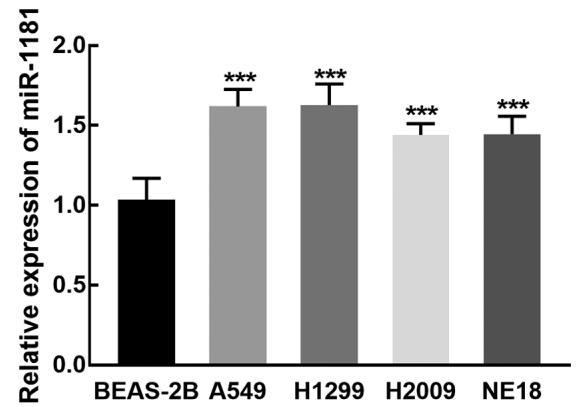

Figure 1. miR-1181 expression in NSCLC tissues and cell lines. (A) miR-1181 was significantly upregulated in NSCLC tissues relative to adjacent non-tumor tissues. (B) miR-1181 was significantly upregulated in NSCLC cell lines (A549, H1299, H2009 and NE18) relative to a normal cell line (BEAS-2B). ${ }^{* * *} \mathrm{P}<0.001$ vs. normal. miR, microRNA; NSCLC, non-small cell lung cancer.

the mean \pm standard deviation obtained from $\geq 3$ experiments. Significant differences between groups were determined by a paired Student's t-test or one-way ANOVA followed by Tukey's post hoc test. The $\chi^{2}$ test was used to evaluate the association between miR-1181 expression and the clinical features of patients. The prognostic value of miR-1181 expression was assessed by Kaplan-Meier followed by long-rank test and Cox regression analysis. $\mathrm{P}<0.05$ was considered to indicate statistical significance.

\section{Results}

miR-1181 is significantly upregulated in NSCLC and associated with lymph node metastasis and TNM stage. The recruited patients were consisted of 69 males and 49 females with an average age of $56.3 \pm 9.33$ years. The expression of miR-1181 was significantly increased in NSCLC tissues compared with that in adjacent normal tissues $(\mathrm{P}<0.001$, Fig. 1A). This upregulation of miR-1181 was also observed in NSCLC cells compared with that in the normal BEAS-2B cells $(\mathrm{P}<0.001$, Fig. 1B).

Using the mean relative miR-1181 expression levels in NSCLC tissues (0.939) as the cut-off, patients with NSCLC were divided into the low and high miR-1181 expression groups. The expression level of miR-1181 was significantly associated with lymph node metastasis $(\mathrm{P}=0.025)$ and TNM stage of patients $(\mathrm{P}=0.013)$, but no significant relationship with any of the other clinical features was identified $(\mathrm{P}>0.05$, Table I).

High expression of miR-1181 is associated with poor prognosis. The survival information of patients with NSCLC was obtained and data were plotted as Kaplan-Meier curves (Fig. 2). The patients with high miR-1181 expression had a poorer prognosis than those with low miR-1181 expression ( $\log$-rank $\mathrm{P}=0.015)$. To further assess the prognostic value of miR-1181, Cox regression analysis was used to determine the influence of miR-1181 expression on the survival probability of the patients. miR-1181 was significantly associated with the survival of patients with NSCLC, with a hazard ratio (HR) of $2.169(95 \% \mathrm{CI}=1.101-4.272, \mathrm{P}=0.025)$. In addition to the TNM stage $(\mathrm{HR}=2.014,95 \% \mathrm{CI}=1.060-3.828$, $\mathrm{P}=0.033$ ), miR-1181 expression in tumor tissues may be considered as an independent prognostic indicator for NSCLC (Table II).
Overexpression of miR-1181 promotes NSCLC cell proliferation. miR-1181 mimics, miR-1181 inhibitor or the corresponding NC were transfected into A549 and H1299 cells to regulate the expression level of miR-1181. The expression of miR-1181 in A549 and H1299 cells was confirmed to be significantly increased after transfection with miR-1181 mimics and to be reduced in the miR-1181 inhibitor-transfected cells $(\mathrm{P}<0.001$, Fig. 3A). To assess the proliferation of the transfected cells, the CCK8 assay was applied, revealing that the proliferation of NSCLC cells with knockdown of miR-1181 was significantly reduced compared with that of the cells with overexpression of miR-1181 ( $\mathrm{P}<0.05$, Fig. 3B).

Overexpression of miR-1181 promotes migration and invasion of NSCLC cells. To evaluate the role of miR-1181 in NSCLC cell migration and invasion, Transwell assays were performed on A549 and H1299 cells transfected with miR-1181 mimics or inhibitor. The results suggested that the number of migrated cells in the group of NSCLC cells with miR-1181 overexpression was significantly elevated, while that of the NSCLC cells with knockdown of miR-1181 was significantly decreased in comparison with the respective controls $(\mathrm{P}<0.001$, Fig. 4A). Similarly, the invasion of NSCLC cells was inhibited by knockdown of miR-1181 and promoted by overexpression of miR-1181 ( $\mathrm{P}<0.001$, Fig. 4B). Hence, these results indicated the regulatory function of miR-1181 in the migration and invasion of NSCLC cells.

AXIN1 is a direct target of miR-1181. The binding sites between miR-1181 and the 3'UTR of AXIN1 were predicted in silico and are presented in Fig. 5A. This predicted binding interaction was then confirmed with a luciferase reporter assay. The relative luciferase activity of the AXIN1 WT reporter plasmid was inhibited by overexpression of miR-1181 in the miR-1181 mimics group and enhanced by the silencing of miR-1181 in the miR-1181 inhibitor group $(\mathrm{P}<0.001$, Fig. 5B). However, the relative luciferase activity of the AXIN1 MT was not significantly affected by the changes in miR-1181 expression ( $\mathrm{P}>0.05$, Fig. 5B).

\section{Discussion}

NSCLC remains the leading cause of cancer-related death with a low 5-year survival rate due to the lack of effective diagnostic methods and therapeutic targets (19). miRNAs have 
Table I. Association between miR-1181 expression and clinical features of patients with non-small cell lung cancer.

\begin{tabular}{|c|c|c|c|c|}
\hline \multirow[b]{2}{*}{ Parameter } & \multirow[b]{2}{*}{ Total patients $(n=118)$} & \multicolumn{2}{|c|}{ Expression of miR-1181 } & \multirow[b]{2}{*}{ P-value } \\
\hline & & High $(n=67)$ & Low $(n=51)$ & \\
\hline Age (years) & & & & 0.509 \\
\hline$<60$ & 63 & 35 & 28 & \\
\hline$\geq 60$ & 55 & 32 & 23 & \\
\hline $\operatorname{Sex}$ & & & & 0.492 \\
\hline Male & 69 & 39 & 30 & \\
\hline Female & 49 & 28 & 21 & \\
\hline Tumor size (cm) & & & & 0.172 \\
\hline$<4$ & 64 & 33 & 31 & \\
\hline$\geq 4$ & 54 & 34 & 20 & \\
\hline Differentiation & & & & 0.102 \\
\hline Well+moderate & 71 & 30 & 41 & \\
\hline Poor & 47 & 37 & 10 & \\
\hline Lymph node metastasis & & & & 0.025 \\
\hline Negative & 67 & 38 & 29 & \\
\hline Positive & 51 & 29 & 22 & \\
\hline TNM stage & & & & 0.013 \\
\hline $\mathrm{I} / \mathrm{II}$ & 68 & 42 & 26 & \\
\hline III/IV & 50 & 25 & 25 & \\
\hline
\end{tabular}

miR, microRNA.

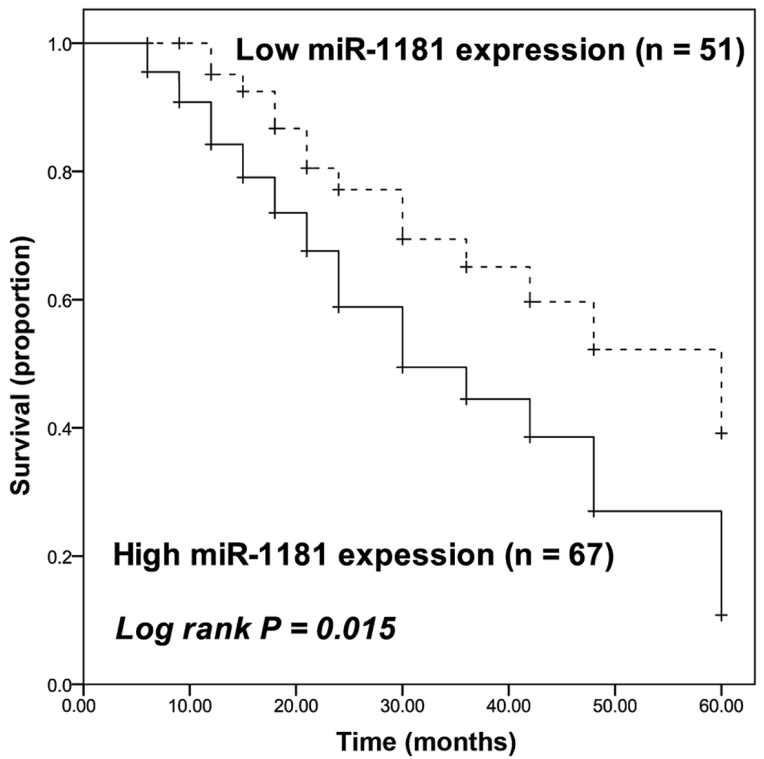

Figure 2. Kaplan-Meier survival curves depicting the association of miR-1181 expression with survival of patients with non-small cell lung cancer. Patients with high miR-1181 expression had a poorer prognosis than those with low miR-1181 expression. Log-rank $\mathrm{P}=0.015$. miR, microRNA.

been indicated to be critical regulators in the pathogenesis of cancer, as certain miRNAs have critical roles in the tumorigenesis and development of various cancer types $(20,21)$. For instance, the expression of miR-21 was significantly associated with the progression of hepatocellular carcinoma (22). In addition, miR-182 promotes the progression of prostate cancer by activating the Wnt/ $\beta$-catenin signaling pathway (23). In NSCLC, miR-675 was reported to enhance the disease progression by activating the $\mathrm{NF}-\kappa \mathrm{B}$ signaling pathway (24) and miR-125a-5p suppressed NSCLC progression by targeting suppressor of variegation 3-9 homolog 1, as the overexpression of miR-125a-5p inhibited cell proliferation, migration and invasion and activated cell apoptosis in NSCLC (25).

A previous study focusing on the miRNA expression profiling of NSCLC indicated that miR-1181 was differentially expressed in NSCLC (12), and thus, it was speculated in the present study that miR-1181 participates in the progression of NSCLC. In the present study, data derived from tissues of patients with NSCLC and NSCLC cell lines suggested significant upregulation of miR-1181, consistent with the previous study. Furthermore, the upregulation of miR-1181 exhibited a significant association with lymph node metastasis and TNM stage in patients with NSCLC. miR-1181 was considered to be able to predict the prognosis of NSCLC, as its upregulation was associated with a poorer survival rate of patients with NSCLC. Accumulating evidence has demonstrated the prognostic value of miRNAs in several cancer types (26-28), and a variety of miRNAs, such as miR-519a, miR-378 and miR-1246, have been considered as prognostic biomarkers for NSCLC (29-31). In the present study, the results of the Cox regression analysis indicated that miR-1181 and the TNM stage were independent prognostic indicators for NSCLC. These results indicated that miR-1181 may be involved in the 
Table II. Multivariate Cox regression analysis between miR-1181 expression and survival rate of patients with non-small cell lung cancer after adjusting for the cofounding factors.

\begin{tabular}{lccr}
\hline Factor & Hazards ratio & 95\% confidence interval & P-value \\
\hline miR-1181 (high vs. low) & 2.169 & $1.101-4.272$ & 0.025 \\
Age ( $\geq 60$ vs. $<60$ ) & 1.134 & $0.640-2.010$ & 0.665 \\
Sex (male vs. female) & 1.185 & $0.657-2.136$ & 0.572 \\
Tumor size ( $\geq 4$ cm vs. $<4$ cm) & 1.572 & $0.827-2.989$ & 0.167 \\
Differentiation (poor vs. well+moderate) & 1.660 & $0.875-3.151$ & 0.121 \\
Lymph node metastasis (positive vs. negative) & 1.670 & $0.884-3.156$ & 0.114 \\
TNM stage (III-IV vs. I-II) & 2.014 & $1.060-3.828$ & 0.033 \\
\hline
\end{tabular}

HR, hazard ratio; miR, microRNA.
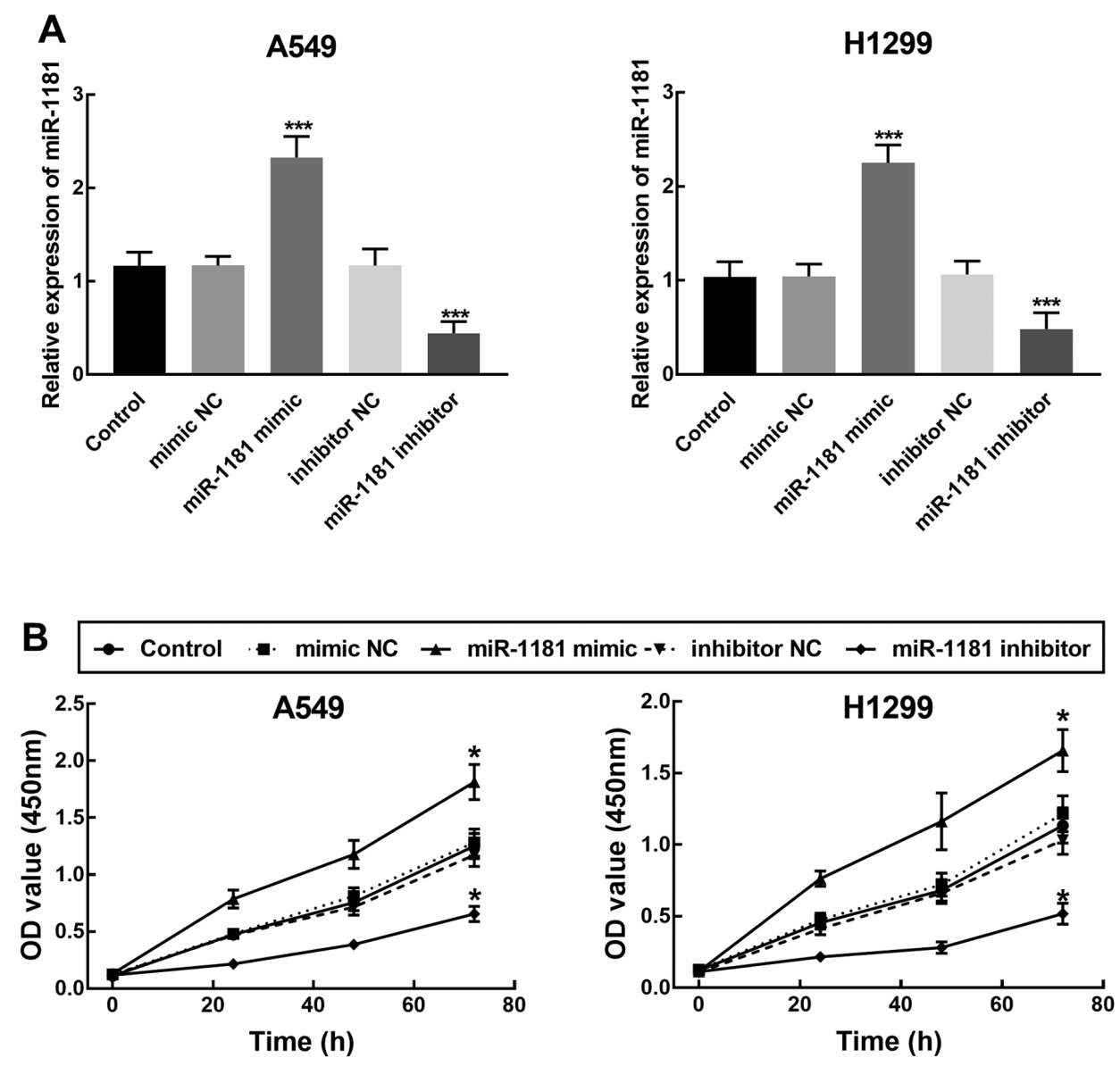

Figure 3. Cell transfection efficiency and effect of miR-1181 expression regulated by cell transfection on the proliferation of A549 and H1299 cells evaluated by the Cell Counting Kit-8 assay. (A) Transfection of miR-1181 mimics significantly increased the expression of miR-1181, while transfection of miR-1181 inhibitor significantly reduced the expression of miR-1181. ${ }^{* * *} \mathrm{P}<0.001$ vs. Control. (B) Overexpression of miR-1181 significantly promoted the proliferation of A549 and H1299 cells, while knockdown of miR-1181 significantly inhibited the proliferation of the non-small cell lung cancer cells. "P<0.05 vs. Control. miR, microRNA; OD, optical density; NC, negative control.

progression of NSCLC and predict poor prognosis of affected patients.

miR-1181 has been reported to be differentially expressed in various cancer types. In hepatocellular carcinoma, miR-1181 was indicated to be upregulated and serve as an oncogene due to its positive effect on the progression of hepatocellular carcinoma by repressing AXIN1 (15). miR-1181 inhibited the migration, invasion and proliferation of pancreatic cancer by targeting STAT3 and inhibited stem cell-like phenotypes by downregulating SOX2 and STAT3 in pancreatic cancer $(13,32)$. A previous study also reported that miR-1181 promoted mesenchymal-epithelial transition in ovarian cancer cells, which is closely associated with the migration and invasion of cancer cells (14). The present results indicated that the miR-1181 mimics increased the expression of miR-1181, which promoted cell proliferation, migration and invasion of NSCLC 

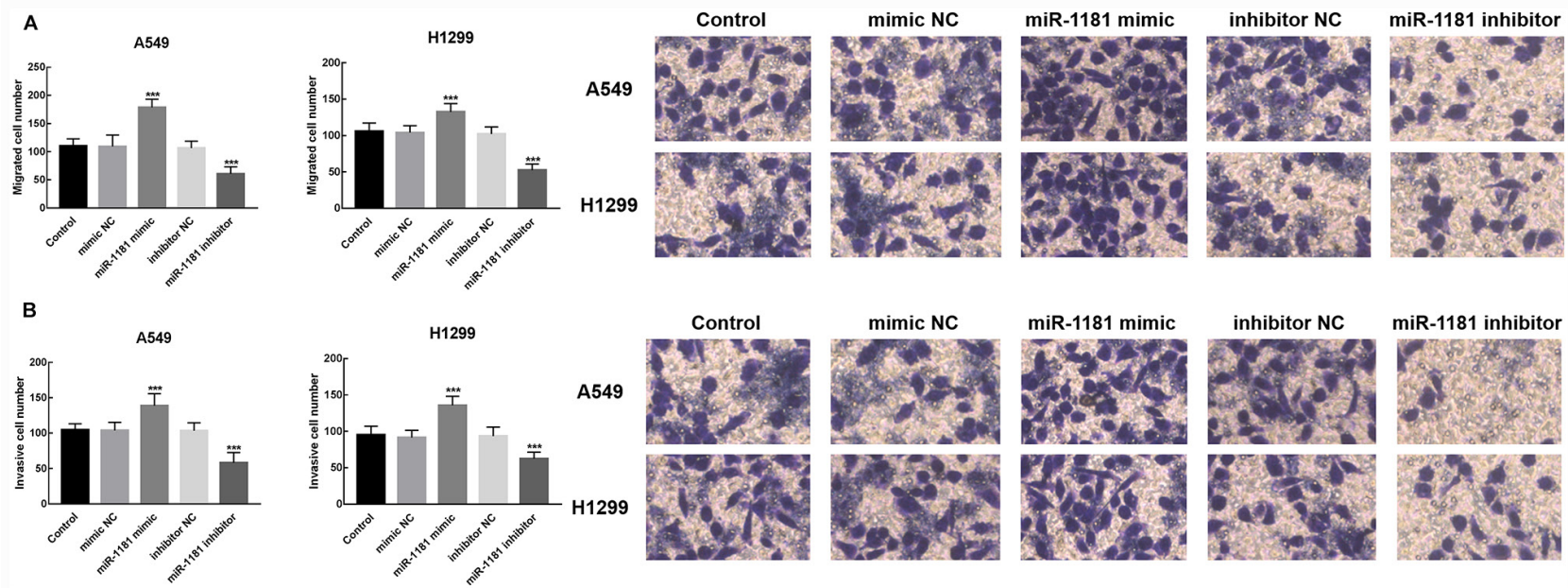

Figure 4. Effect of miR-1181 expression on the migration and invasion of A549 and H1299 cells evaluated by Transwell assay. (A) Migration and (B) invasion of A549 and H1299 cells was promoted by overexpression of miR-1181 and inhibited by knockdown of miR-1181. Magnification, $\mathrm{x} 400$. ${ }^{* * *} \mathrm{P}<0.001 \mathrm{vs}$. Control. miR, microRNA; NC, negative control.

A

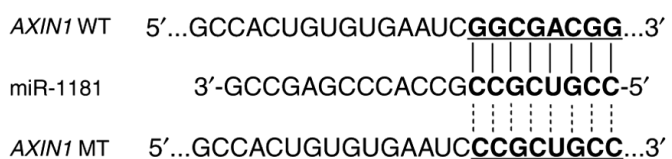

B

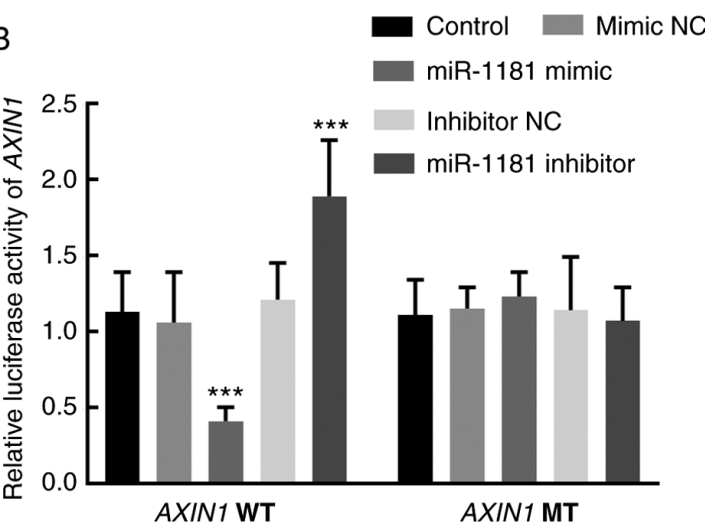

Figure 5. Targeting interaction of miR-1181 and AXIN1. (A) The binding sites of AXIN1 3'-untranslated region and miR-1181. (B) miR-1181 mimics significantly inhibited the relative luciferase activity of the AXIN1 WT reporter plasmid, while knockdown of miR-1181 significantly enhanced the relative luciferase activity of the AXIN1 WT reporter plasmid. However, the relative luciferase activity of the AXIN1 MT plasmid was not affected by the expression of miR-1181. ${ }^{* * *} \mathrm{P}<0.001$ vs. Control. miR, microRNA; WT, wild-type; MT, mutant; NC, negative control; AXIN1, axin 1.

cells, while miR-1181 inhibitor reduced miR-1181 expression and decreased cell proliferation, migration and invasion. These results demonstrated that miR-1181 may serve as a biomarker for the progression and prognosis of NSCLC.

Previously, AXIN1 was reported to be a specific target of miR-1181 during the regulation of miR-1181 in hepatocellular carcinoma (15). It was speculated that the tumor promoter role of miR-1181 was a result of targeting AXIN1. The results of the luciferase reporter assay of the present study suggested that overexpression of miR-1181 significantly inhibited the relative luciferase activity of the AXIN1 WT reporter plasmid, while it was enhanced by knockdown of miR-1181. These results indicated that AXIN1 is a direct target of miR-1181 with involvement in the progression of NSCLC.

However, more work is still required in future studies. In vivo experiments are important parts in the investigation of tumor development $(33,34)$. The results of the present study revealed the tumor promoter role of miR-1181 in NSCLC in vitro, while in vivo experiments, such as in vivo migration and invasion assays and tumor growth assays, are also needed to verify the function of miR-1181 in the progression of NSCLC.

In conclusion, the present study indicated that the upregulation of miR-1181 in NSCLC tissues was associated with lymph node metastasis and TNM stage of patients and predicted poor prognosis. miR-1181 may be involved in the progression of NSCLC due to enhancing the proliferation, migration and invasion of NSCLC cells. These results indicated that miR-1181 may serve as a potential novel therapeutic target for the treatment of NSCLC.

\section{Acknowledgements}

Not applicable.

\section{Funding}

No funding was received.

\section{Availability of data and materials}

The datasets used and/or analyzed during the current study are available from the corresponding author on reasonable request.

\section{Authors' contributions}

FL, GM, BW, XW and CH conceived the study. FL GM, and BW performed the experiments and wrote the manuscript. FL, $\mathrm{XW}$ and $\mathrm{CH}$ analyzed the data. All authors read and approved the final version of the manuscript. FL and GM checked and approved the authenticity of the raw data. 


\section{Ethics approval and consent to participate}

This study was approved by the Ethics Committee of Nanjing Chest Hospital (Nanjing, China). All patients provided written informed consent.

\section{Patient consent for publication}

Not applicable.

\section{Competing interests}

The authors declare that they have no competing interests.

\section{References}

1. Parkin DM, Bray FI and Devesa SS: Cancer burden in the year 2000. The global picture. Eur J Cancer 37 (Suppl 8): S4-S66, 2001.

2. Bray F, Ferlay J, Soerjomataram I, Siegel RL, Torre LA and Jemal A: Global cancer statistics 2018: GLOBOCAN estimates of incidence and mortality worldwide for 36 cancers in 185 countries. CA Cancer J Clin 68: 394-424, 2018.

3. Bilfinger T, Keresztes R, Albano D and Nemesure B: Five-year survival among stage IIIA lung cancer patients receiving two different treatment modalities. Med Sci Monit 22: 2589-2594, 2016.

4. Siegel RL, Miller KD and Jemal A: Cancer statistics, 2018. CA Cancer J Clin 68: 7-30, 2018.

5. Minna JD, Roth JA and Gazdar AF: Focus on lung cancer. Cancer Cell 1: 49-52, 2002.

6. Lin S and Gregory RI: MicroRNA biogenesis pathways in cancer. Nat Rev Cancer 15: 321-333, 2015.

7. Yang J, Zhang Z, Chen S, Dou W, Xie R and Gao J: miR-654-3p predicts the prognosis of hepatocellular carcinoma and inhibits the proliferation, migration, and invasion of cancer cells. Cancer Biomark 28: 73-79, 2020

8. Wang M, Qiu R, Gong Z, Zhao X, Wang T, Zhou L, Lu W, Shen B, Zhu W and $\mathrm{Xu} \mathrm{W}$ : miR-188-5p emerges as an oncomiRNA to promote gastric cancer cell proliferation and migration via upregulation of SALL4. J Cell Biochem 120: 15027-15037, 2019.

9. Tang X, Ding Y, Wang X, Wang X, Zhao L and Bi H: miR-650 promotes non-small cell lung cancer cell proliferation and invasion by targeting ING4 through Wnt- $1 / \beta$-catenin pathway. Oncol Lett 18: 4621-4628, 2019.

10. Luo J, Pan J, Jin Y, Li M and Chen M: miR-195-5p Inhibits proliferation and induces apoptosis of non-small cell lung cancer cells by targeting CEP55. Onco Targets Ther 12: 11465-11474, 2019.

11. Zhong K, Chen K, Han L and Li B: MicroRNA-30b/c inhibits non-small cell lung cancer cell proliferation by targeting Rab18. BMC Cancer 14: 703, 2014.

12. Hu Y, Wang L, Gu J, Qu K and Wang Y: Identification of microRNA differentially expressed in three subtypes of non-small cell lung cancer and in silico functional analysis. Oncotarget 8: 74554-74566, 2017.

13. Wang J, Guo XJ, Ding YM and Jiang JX: miR-1181 inhibits invasion and proliferation via STAT3 in pancreatic cancer. World J Gastroenterol 23: 1594-1601, 2017.

14. Zhang HY, Li JH, Li G and Wang SR: Activation of ARK5/miR-1181/HOXA10 axis promotes epithelial-mesenchymal transition in ovarian cancer. Oncol Rep 34: 1193-1202, 2015.

15. Song Z, Yu Z, Chen L, Zhou Z, Zou Q and Liu Y: MicroRNA-1181 supports the growth of hepatocellular carcinoma by repressing AXIN1. Biomed Pharmacother 119: 109397, 2019.
16. Livak KJ and Schmittgen TD: Analysis of relative gene expression data using real-time quantitative PCR and the 2(-Delta Delta C(T)) method. Methods 25: 402-408, 2001.

17. Cai Z, Li J, Zhuang Q, Zhang X, Yuan A, Shen L, Kang K, Qu B, Tang Y, Pu J, et al: MiR-125a-5p ameliorates monocrotalineinduced pulmonary arterial hypertension by targeting the TGF- $\beta 1$ and IL-6/STAT3 signaling pathways. Exp Mol Med 50: 1-11, 2018.

18. Benderska N, Dittrich AL, Knaup S, Rau TT, Neufert C, Wach S, Fahlbusch FB, Rauh M, Wirtz RM, Agaimy A, et al: miRNA-26b overexpression in ulcerative colitis-associated carcinogenesis. Inflamm Bowel Dis 21: 2039-2051, 2015.

19. Pao W: New approaches to targeted therapy in lung cancer. Proc Am Thorac Soc 9: 72-73, 2012.

20. Liu CG, Calin GA, Meloon B, Gamliel N, Sevignani C, Ferracin M, Dumitru CD, Shimizu M, Zupo S, Dono M, et al: An oligonucleotide microchip for genome-wide microRNA profiling in human and mouse tissues. Proc Natl Acad Sci USA 101: 9740-9744, 2004.

21. Volinia S, Calin GA, Liu CG, Ambs S, Cimmino A, Petrocca F, Visone R, Iorio M, Roldo C, Ferracin M, et al: A microRNA expression signature of human solid tumors defines cancer gene targets. Proc Natl Acad Sci USA 103: 2257-2261, 2006.

22. Yoon JS, Kim G, Lee YR, Park SY, Tak WY, Kweon YO, Park JG, Lee HW, Han YS, Ha HT, et al: Clinical significance of microRNA-21 expression in disease progression of patients with hepatocellular carcinoma. Biomark Med 12: 1105-1114, 2018.

23. Wang D, Lu G, Shao Y and Xu D: MiR-182 promotes prostate cancer progression through activating Wnt $/ \beta$-catenin signal pathway. Biomed Pharmacother 99: 334-339, 2018.

24. Feng Y, Yang C, Hu D, Wang X and Liu X: miR-675 promotes disease progression of non-small cell lung cancer via activating NF- $\kappa$ B signaling pathway. Cell Mol Biol (Noisy-le-grand) 63: 7-10, 2017.

25. Liu H, Ma Y, Liu C, Li P and Yu T: Reduced miR-125a-5p level in non-small-cell lung cancer is associated with tumour progression. Open Biol 8: 180118, 2018.

26. Shao C, Yang F, Qin Z, Jing X, Shu Y and Shen H: The value of miR-155 as a biomarker for the diagnosis and prognosis of lung cancer: A systematic review with meta-analysis. BMC Cancer 19: 1103, 2019

27. An JX, MaZS, Ma MH, Shao S, Cao FL and Dai DQ: MiR-1236-3p serves as a new diagnostic and prognostic biomarker for gastric cancer. Cancer Biomark 25: 127-132, 2019.

28. Peng X, Pan X, Liu K, Zhang C, Zhao L, Li H, Guan X, Xu W, Xu J, Zhang F and Lai Y: miR-142-3p as a novel biomarker for predicting poor prognosis in renal cell carcinoma patients after surgery. Int J Biol Markers 34: 302-308, 2019.

29. Wang Y, Jiang F, Wang J, Fu Y, Li Y and Li F: MiR-519a functions as a tumor suppressor and is negatively associated with poor prognosis of non-small cell lung cancer. Cancer Biomark 28: 121-128, 2020.

30. Zhang Y and Xu H: Serum exosomal miR-378 upregulation is associated with poor prognosis in non-small-cell lung cancer patients. J Clin Lab Anal 34: e23237, 2020.

31. Fan L, Wang J, Cao Q, Ding X and Li B: Aberrant miR-1246 expression promotes radioresistance in non-small cell lung cancer: A potential prognostic biomarker and radiotherapy sensitization target. Am J Cancer Res 10: 314-335, 2020.

32. Jiang J, Li Z, Yu C, Chen M, Tian S and Sun C: MiR-1181 inhibits stem cell-like phenotypes and suppresses SOX2 and STAT3 in human pancreatic cancer. Cancer Lett 356: 962-970, 2015.

33. Ning L, Zhang M, Zhu Q, Hao F, Shen W and Chen D: miR-25-3p inhibition impairs tumorigenesis and invasion in gastric cancer cells in vitro and in vivo. Bioengineered 11: 81-90, 2020.

34. Soheilyfar S, Velashjerdi Z, Sayed Hajizadeh Y, Fathi Maroufi N, Amini Z, Khorrami A, Haj Azimian S, Isazadeh A, Taefehshokr S and Taefehshokr N: In vivo and in vitro impact of miR-31 and miR-143 on the suppression of metastasis and invasion in breast cancer. J BUON 23: 1290-1296, 2018. 